\title{
Coevaluación de competencias a través de la metodología del caso en el Grado en Administración y Dirección de Empresas Coevaluation of competencies using the case study method in the Bachelor's Degree in Business Administration
}

\author{
Harold Torrez Meruvia, Sergio Cruz Almanchel, Mariona Vilà Bonilla \\ harold.torrez@eae.es, sergio.cruz@eae.es,mvila@eae.es \\ Grupo de Innovación Docente (GID-EAE) \\ EAE Business School \\ Barcelona, España
}

\begin{abstract}
Resumen- La implantación del Espacio Europeo de Educación Superior ha supuesto que las universidades a nivel europeo desarrollen metodologías y acciones que fomenten la adquisición de competencias. La investigación desarrollada en una asignatura de tercer curso de grado en Administración y Dirección de Empresas permite medir el nivel de la percepción de competencias que adquieren los estudiantes a medida que van resolviendo diversos casos de estudio. Es por ello que, para generar un componente de objetividad en la medición del nivel de percepción de sus competencias, se ha visto conveniente realizar una prueba en dos vertientes diferenciadas. Por una parte, la autoevaluación y por otra, la evaluación del profesor para conocer el nivel de competencias que van adquiriendo los estudiantes. Se puede apreciar que los resultados obtenidos demuestran que existe un nivel similar entre la percepción que tienen los estudiantes y el profesor, lo cual brinda un nivel de confianza ya que ambos participantes están midiendo las competencias homogéneamente.
\end{abstract}

\section{Palabras clave: Competencias, Autoevaluación, Coevaluación}

Abstract- With the implantation of the European Higher Education Area, European universities have developed methodologies and actions that foster the acquirement of competencies. Research carried out in a third year course of the Bachelor's Degree in Business Administration allows for the measurement of the level of perceived competency acquirement by the students, as they solve different case studies. In order to generate a more objective measurement of the levels of perceived competency acquirement, this study notes the suitability of realizing tests based on two different aspects. On the one hand, self-evaluation and on the other hand, the evaluation of the professor in order to know the level of competency acquirement of their students. The results obtained make apparent a similarity between the perception of competency acquirement of the students and the professor. This provides a level of confidence, given that the competencies are measured homogenously by all participants.

Keywords: Competencies, self-evaluation, coevaluation

\section{INTRODUCCIÓN}

Uno de los primeros investigadores en definir y aplicar el concepto de competencia fue White (1959), que definió de forma explícita una competencia como la capacidad que tiene una persona para interactuar con su entorno. Las competencias tienen en sí mismas un aspecto de motivación y no hacen referencia a una capacidad instintiva.

McClelland (1973) remarcó la importancia de este término, poniendo en cuestión la validez de los test convencionales de inteligencia y aptitud, ya que afirmaba que estos test no mostraban aquellas habilidades que serían necesarias en el mundo laboral.

Propuso un test para valorar competencias como una alternativa a los test de inteligencia y dio algunas indicaciones de cómo hacer esta valoración. Este trabajo se ha convertido en la referencia clásica relativa a la introducción de las competencias en el área de recursos humanos, tanto para la selección de personas como para su gestión y su evaluación en el ámbito laboral. Spencer y Spencer (1993) completaron la propuesta genérica de McClelland con un "diccionario de competencias" universales, determinadas a partir de entrevistas realizadas sobre 200 puestos de trabajo. También definieron competencia como "una característica personal latente que mantiene una relación causal con un criterio de desarrollo eficaz o superior en un trabajo o situación”.

Levy-Leboyer (1997) afirma que existen tres formas de desarrollar las propias competencias: antes de la vida profesional mediante la formación previa, durante la vida activa a través de cursos de formación para adultos y por el ejercicio mismo de una actividad profesional.

Conviene destacar que en la adquisición de competencias la experiencia juega un papel muy importante. Las competencias tienen entonces un carácter muy dinámico, ya que se desarrollan al lado de las experiencias profesionales, y éstas pueden ser adquiridas a lo largo de toda la vida activa. Además, las competencias dependen de las experiencias específicas y están más ligadas a un sector profesional, a una empresa, a un lugar o incluso a un momento determinado.

Por este motivo, las empresas tienen interés en conocer las competencias concretas que caracterizan a sus recursos humanos. La adquisición de una competencia se presenta como un proceso escalonado que llega a su cumbre cuando se está desarrollando la actividad profesional en un puesto, una empresa y un entorno concreto. Entendiéndose de esta forma que, pese a la posible formación previa, el desarrollo de una 
competencia propiamente sólo es posible cuando se añade el factor experiencia (mediante la actividad profesional). Las aptitudes y los rasgos de personalidad permiten caracterizar a los individuos y explicar la variación de sus comportamientos en la ejecución de tareas específicas, mientras que las competencias afectan de manera integral a las aptitudes, rasgos de personalidad y conocimientos adquiridos, para cumplir correctamente con una misión compleja.

Dubois et al. (2004) señalaron que debe utilizarse el concepto de competencia y su amplia gama de definiciones para poder describir de forma fidedigna el comportamiento, las motivaciones y el conocimiento relacionado con el éxito o el fracaso de la vida laboral. En la actualidad hay dos escuelas de pensamiento que hacen referencia a la interpretación del concepto de competencia: la primera mantiene que una competencia implica conocimiento o habilidades, mientras la segunda interpreta una competencia como cualquier otra característica que apoya la actividad. En esta segunda interpretación, una competencia puede incluir conocimientos, habilidades, u otras características como el nivel de motivación o rasgos personales.

En resumen, las competencias pueden entenderse como una combinación de atributos de un trabajador, este trabajador debe haber producido previamente una adecuada asimilación e integración de su formación, experiencia y habilidades. Para comprender el perfil y el nivel óptimo de competencias en un trabajo y en una organización concreta, se debe centrar el análisis en los comportamientos observables de las personas consideradas de alto rendimiento en ese ámbito según indica Blanco (2007). Por otra parte, el desarrollo de las competencias parece que necesita de la experiencia adquirida en la actividad profesional concreta dentro de la empresa, como indican Levy-Leboyer (1997), pero sin dejar a un lado la formación académica, donde las personas inician su desarrollo a través de una serie de actividades que potencian el despertar de su talento.

\section{CONTEXTO}

La formación universitaria europea actualmente está enfocada en el futuro mundo laboral de los estudiantes; esto ha fomentado la aparición de una preocupación creciente en cuestiones relacionadas con la mejora de la calidad docente, véase Zabalza (2004).

¿Se pueden adquirir estas competencias desde la formación universitaria? ¿Se pueden enseñar las competencias en el aula? Si "las competencias profesionales se identifican en función de un puesto de trabajo de un perfil profesional”, se hace difícil evaluar competencias en la universidad. Esto es así porque las competencias no se adquieren en el entorno laboral real, puesto que "las competencias no pueden evaluarse plenamente hasta que se utilicen en un determinado puesto de trabajo", tal y como señala Oliveros (2007).

Pese a la complejidad del proceso de desarrollo de una competencia, para Oliveros parece ser un proceso progresivo dividido en dos fases: primero, la formación de competencias basada en la actividad desarrollada en el aula y en la que el individuo no ejercita la conducta pero se trabajan algunos de sus aspectos y componentes; y segundo, el desarrollo en el puesto de trabajo que consiste en ubicar al individuo en una situación que implique practicar una determinada conducta. También afirma, los estudiantes deben empezar a practicar las conductas técnicas en las aulas de la universidad, en las actividades fuera de estas aulas y en los centros de prácticas.

El desarrollo de las competencias transversales debe incluirse en el conjunto de titulaciones universitarias, concibiendo su desarrollo como una continuación del iniciado en anteriores niveles educativos, que posteriormente seguirá desarrollándose en el ejercicio profesional.

Por las razones anteriores, las universidades se esfuerzan por orientar los programas al mundo profesional mediante prácticas en empresa o casos prácticos para que los estudiantes puedan ver la situación real.

Martínez et al. (2010) ponen de manifiesto que en el sistema educativo español actual cada vez toma más importancia la preocupación por el nivel de desajuste entre las competencias potenciadas por la universidad y el perfil de competencias demandado por las empresas. Entre los resultados obtenidos puede destacarse que existen grandes diferencias entre el desarrollo alcanzado por los estudiantes y los requerimientos por parte de la empresa (déficits) en lo que se refiere a las competencias genéricas de escucha, debate y argumentación, el sentido crítico y autocrítico y la orientación al aprendizaje. En cambio puede apreciarse un exceso de capacidad en lo que hace referencia a la autonomía e iniciativa, la aplicación de conceptos teóricos y el compromiso ético. Según los estudiantes, la universidad presenta déficits en competencias como la inteligencia emocional, escucha, debate y argumentación y gestión del tiempo. En cambio, otorga más importancia de la exigida por el mercado laboral en trabajo en equipo y aplicación de conceptos. Según los profesores, la universidad presenta déficits en aplicación de términos analíticos, orientación al aprendizaje y el sentido ético. Y ofrece más formación competencial de la requerida en la utilización de las TIC. Se hace evidente que las percepciones son diferentes.

En este sentido, el objetivo que se pretende con este trabajo es conocer la percepción del nivel de competencias que los estudiantes creen desarrollar al realizar cada una de las actividades prácticas dentro la asignatura, mediante un proceso de autoevaluación y coevaluación.

\section{DESCRIPCIÓN}

Las competencias seleccionadas de la asignatura de tercer curso "Desarrollo profesional III" se han establecido en la memoria de la titulación del Grado en Administración y Dirección de Empresas (ADE) y se detallan en la tabla 1.

Tabla 1. Lista de competencias

- Comunicación oral y escrita

- Trabajar equipo interdisciplinar

- Gestionar datos

- Conocer las limitaciones del conocimiento y las competencias

- Conocer los obstáculos y oportunidades del entorno

- Entorno social

- Análisis de problemas

- Entender la organización de empresa 
Estas competencias son obligatorias y deben motivarse y evaluarse durante el curso académico. Esto es necesario para cumplir con los objetivos competenciales que tiene la asignatura, y se realiza a través de la aplicación de la metodología del caso y la resolución de problemas reales.

Tabla 2: Lista de casos trabajados en la asignatura.

Actividad 1: Caso 100 montaditos

Actividad 2: Caso Lego

Actividad3: Facebook

Actividad 4: Google

Actividad 5: Hoteles Marriott

Actividad 6: Camper

Para la aplicación, se pide a los estudiantes que formen grupos de trabajo de entre 4 y 6 personas y que resuelvan un caso empresarial en función de los conocimientos que han adquirido. Para ello, se entrega un documento con las instrucciones a seguir y el caso a resolver a cada uno de los grupos. Los estudiantes se reúnen y estudian la situación en función de los contenidos desarrollados en la asignatura, para finalmente proponer una estrategia a seguir.

A continuación, se pide que los estudiantes realicen una autoevaluación de las competencias que creen haber desarrollado y aplicado en el caso presentado en una escala del 0 al 10. Esto se evalúa mediante una encuesta tanto para los estudiantes como para el profesor. Esta encuesta se compone por ocho preguntas, cada una de ellas hace referencia a una de las competencias indicadas en la tabla 1.

La evaluación del profesor se lleva a cabo a través de dos acciones: por una parte, la observación durante la realización de la actividad grupal, donde se valora las competencias de comunicación oral y escrita, trabajo en equipo y análisis de problemas de cada estudiante. Por la otra parte, una vez los estudiantes resuelven el caso de estudio, el profesor evalúa la actividad en relación a las competencias restantes.

Desde el ámbito metodológico, el aplicar una estrategia que integre a los estudiantes y al profesor es importante ya que permite tener un nivel de objetividad en el desarrollo de las competencias. Los resultados que se presentan responden a seis casos empresariales que se realizaron en las clases de la asignatura "Desarrollo profesional III", donde se aprecia la autoevaluación y la coevaluación.

\section{Resultados}

Para llevar a cabo la investigación, se aplicó una encuesta de evaluación al profesor y una de autoevaluación a los 43 estudiantes de un curso, donde se hacía referencia a la percepción del nivel de competencias adquirido en cada uno de los seis casos que forman la asignatura.

A continuación, se presentan los resultados obtenidos, correspondientes a la percepción del nivel de competencias, expresado en valores medios, obtenidos por el profesor y por los estudiantes.

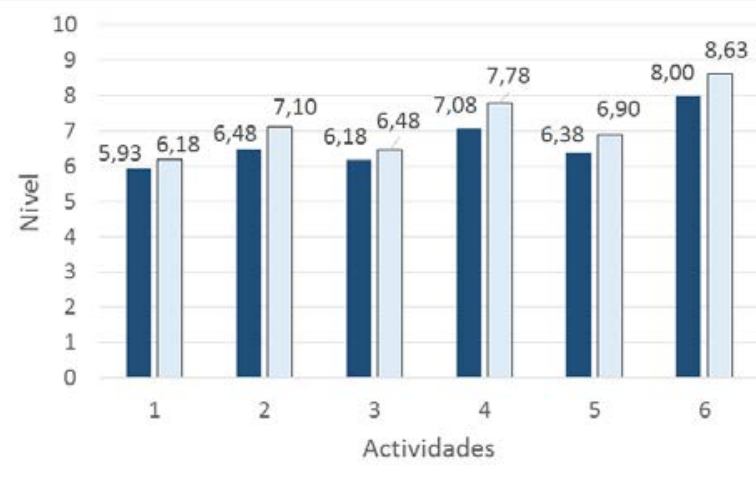

- Autoevaluación del estudiante $\quad \square$ Coevaluación del profesor

Figura 1. Percepción del nivel de competencias adquiridas por los estudiantes, valorado del 0 al 10, en los 6 casos de empresa realizados en clase.

Se observa similitud en cada una de las actividades realizadas: al comparar la valoración del profesor, esta siempre es ligeramente superior que la de los estudiantes. Este comportamiento, justifica y crea veracidad en el estudio realizado, pues muestra una percepción del nivel de competencias homogénea entre las dos partes. Lo cual demuestra que el hecho de aplicar una herramienta a través de dos etapas paralelas, concretamente la autoevaluación y la coevaluación, nos permite obtener información de la percepción de las competencias adquiridas en los estudiantes desde un punto de vista más objetivo y real.

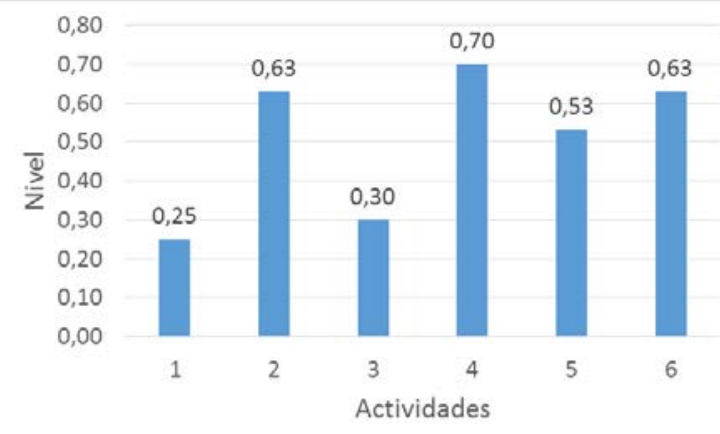

Figura 2. Análisis de las diferencias medias entre la percepción del nivel de competencias adquirido según el profesor y los alumnos.

En detalle, podemos ver el análisis de las diferencias, donde encontramos las mayores en las actividades 2, 4 y 6, con valores de 0,63 y 0,7. Este hecho puede deberse a una mayor complejidad de las actividades y un mayor tiempo necesario para desarrollarlas. Esto quiere decir que el profesor esperaba que los estudiantes realizaran un trabajo con un mayor grado de síntesis, presentación de resultados o reflexión.

Si realizamos un análisis a nivel más general, tomando como referencia la media de las diferencias, obtenemos un valor de 0,5. Esto quiere decir que el profesor ha tenido una percepción media superior a la del alumno en media fracción. Esto indica que el profesor ve que el estudiante ha desarrollado las competencias de mejor manera, mientras que el estudiante demuestra una cierta inseguridad, claramente reflejada en la valoración, y en estrecha relación con el autodiagnóstico y autoconocimiento del estudiante. 


\section{CONCLUSIONES}

El desarrollo de la asignatura en el tercer curso es un momento fundamental para motivar la consolidación de competencias, es por ello que a nivel metodológico la aplicación de casos prácticos reales permiten que el estudiante pueda poner en práctica una serie de habilidades diferenciales que se asemejan a las que puede realizar en un entorno empresarial en el futuro.

La autoevaluación del nivel de competencias adquiridas por los propios estudiantes les ayuda a conocerse, lo cual puede ser un factor positivo en su empleabilidad. Los resultados de los estudiantes muestran claramente que ellos sienten que sus capacidades y habilidades se han visto plenamente incrementadas en cada una de las actividades a lo largo del curso académico, lo cual les da cierta seguridad de responder positivamente a la encuesta. La coevaluación que realiza el profesor consolida la percepción de los estudiantes, por lo tanto aporta fiabilidad al proceso de autoevaluación.

La metodología descrita en este trabajo permite establecer un ciclo de mejora continua en el desarrollo de las competencias en los estudiantes. Dado el carácter práctico de esta asignatura, podemos diseñar nuevas actividades, no solo de resolución de problemas a nivel empresarial, si no de juegos de rol que permitan imitar el entorno empresarial y de gestión.

\section{REFERENCIAS}

Blanco, A. (2007). Trabajadores competentes: introducción y reflexiones sobre la gestión de recursos humanos por competencias. ESIC Editorial.

Colménter, A. L. C. (2004). El concepto de universidad: origen y evolución. Ediciones del Rectorado.
Dubois, D., \& Rothwell, W. (2004). Competency-Based Human Resource Management: Discover a New System for Unleashing the Productive Power of Exemplary Performers. Nicholas Brealey Publishing.

Dubois, D. D. et al. (2004). Competency-Based Human Resource Management, Davies-black Publishing. Hardcover. 376.

Levy-Leboyer, C. (1997). Gestión de las Competencias: cómo analizarlas, cómo evaluarlas, cómo desarrollarlas, Ediciones Gestión 2000.

Martínez De Ibarreta Zorita, C., San Roque, I. M., Astudillo, M. J. P., López, S. C., Rodriguez, L. F., \& San Román, P. M. (2010). Evaluación del grado de ajuste entre el perfil competencial demandado por las empresas y el obtenido por los estudiantes de ADE. Investigaciones de Economía de la Educación. Volume 5, 5, 245-266.

McClelland, D. C. (1973). Testing for competence rather than for "intelligence". American psychologist, Volume 28(1), 1.

Oliveros, L., 2007. Identificación de competencias: una estrategia para la formación en el Espacio Europeo de Educación Superior. Universidad Complutense de Madrid. Revista Complutense de Educación. Volume 17 (1).

Spencer Jr, L. M., \& Spencer, S. M. (1993). Competence at Work: Models for Superior Performance. John Wiley \& Sons. Nueva York.

White, R. W. (1959). Motivation reconsidered: The concept of competence. Psychological review, 66(5), 297.

Zabalza, M. A. (2004). La enseñanza universitaria. El escenario y sus protagonistas. Madrid, Narcea, S.A. 